\title{
MULTICOLOR SURVEY FOR HIGH REDSHIFT QUASARS BY KISO SCHMIDT TELESCOPE
}

\author{
TOMOHIKO YAMAGATA \\ Elementary and Secondary Education Bureau \\ Ministry of Education, Science, Sports and Culture \\ Kasumigaseki, Chiyoda Tokyo 100, Japan \\ Electric mail: yamagata@omega.mtk.ioa.s.u-tokyo.ac.jp \\ AND \\ KIMIAKI KAWARA, TSUTOMU AOKI AND YOSHIAKI SOFUE \\ Institute of Astronomy, University of Tokyo \\ Mitaka Tokyo 181, Japan \\ Electric mail: kkawara@ioa.s.u-tokyo.ac.jp
}

\section{Introduction}

Several groups have been successful in the survey of high-redshift $(z>4)$ quasars. For example, Schneider et al. (1994) and Schmidt et al. (1995) were successful in detecting 90 quasars with $2.75<z<4.75, R \leq 21$ for $62 \mathrm{deg}^{2}$ using Palomar Transit Grism. Among them, there are 9 quasars with $z>4$.0. Storrie-Lombardi et al. (1996) were detected 31 quasars with $z \geq 4.0$, $m_{r} \leq 19$ for $2500 \mathrm{deg}^{2}$ by APM photographic multicolor survey using UK Schmidt telescope. Survey observations of quasars up to now have to select one of the following conditions; wide field surveys using photographic Schmidt plate which cover large areas but do not go faint enough, or deep surveys using large telescopes which go faint magnitude but which cover relatively small areas.

In this situation, we decided to begin a deep and wide-field multicolor survey for high-redshift quasars using the Kiso Schmidt telescope with a wide-field CCD camera. The Kiso Schmidt telescope has a corrector of $105 \mathrm{~cm}$ in diameter and a $150 \mathrm{~cm}$ main mirror. In order to utilize wide-field of the telescope, wide-field CCD cameras are being developed at the Kiso Observatory. As a first step a new large formatted CCD camera (hereafter referred to as $2 \mathrm{~K}-\mathrm{CCD}$ ) is commissioning in a few month. The CCD chip used in this camera was provided by SITe and has $2048 \times 2048$ pixels which gives us a field of view of $50^{\prime} \times 50^{\prime}$ at the prime focus of the telescope. The coverage of the field is expected to be upgraded soon to a $2^{\circ} \times 2^{\circ}$ with mosaic of $2 \times 4$ CCD chips as a next generation imaging system of the Kiso Observatory. The standard UBVRI Johnson-Cousins color bands are adopted for $2 \mathrm{~K}-\mathrm{CCD}$ system. We modified the standard system dividing $I$ band into $I^{\prime}$ and $Z$ bands shown in Figure $1 \mathrm{~b}$ so as to be appropriate to detect quasars of redshift up to 6.0 (see discussion in section 3). In this paper, we present the result of the test observation of known quasars with the existing CCD camera, and discuss the performance of the survey by simple model of spectral energy distribution (hereafter referred to as SED) of high-redshift quasars.

\section{Observation of Known Quasars}

In order to examine the feasibility of multicolor CCD survey with the Kiso Schmidt telescope, the existing CCD Camera system (hereafter referred to as 1K-CCD) of the Kiso Observatory was used to observe VRI colors of 4 known quasars with redshift $z>4$. The camera was made of Tektronics CCD clip with $1024 \times 1024$ pixels giving a field of view of $12^{\prime} .5 \times 12^{\prime} .5$ with $0.37^{\prime \prime} /$ pixel at the prime focus of the Kiso Schmidt telescope. Figure 1a shows $V R I$ response function for the $1 \mathrm{~K}$-CCD system. We used VRI color bands for the test observation since the Lyman alpha line emitted at $4.0<z<5.0$ is redshifted to $6000 \AA$ or more and drops into the $R$ band. Therefore 

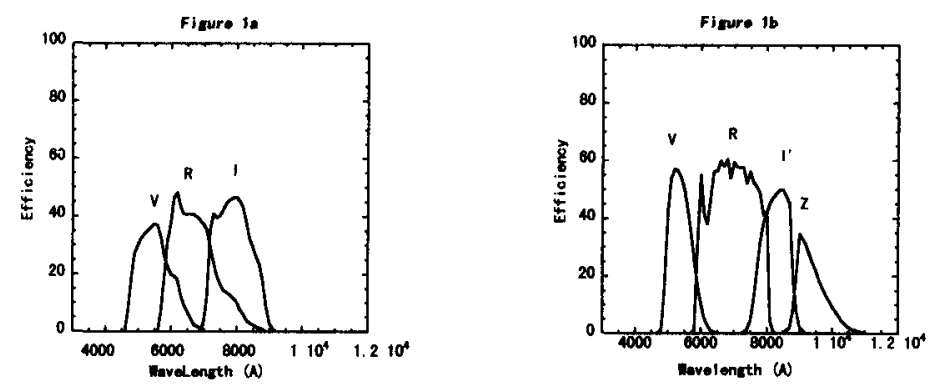

Figure 1. (a) VRI response function for the 1K-CCD system. $R$ band of $1 \mathrm{~K}$-CCD is slightly bluer and narrower than the standard system. (b) $V R I^{\prime} Z$ responce function for modified 2K-CCD system. The standard UBVRI Johnson-Cousins color bands are adopted for original $2 \mathrm{~K}$-CCD system. We modified the standard system dividing $I$ band into $I^{\prime}$ and $Z$ bands so as to be appropriate to detect quasars of redshift up to 6.0 .

quasars with $4.0<z<5.0$ is expected to be distinguished from field stars using 2-color diagram of $(V-R)$ vs. $(R-I)$. Table 1 summarizes the coordinates and redshifts of the observed quasars. We obtained $V R I$ images around 4 quasars. The standard IRAF system was used for flat-fielding and dark-subtracting of CCD data. Source detection and photometry are performed by the automated detection package developed by Yamagata (1986) and Yamagata and Yoshii (1991). The output from the detection package is compared to the CCD image, and then extended sources and artifact such as cosmic ray hits are removed. The sky brightness is assumed to be $21 \mathrm{mag} / \mathrm{deg}^{2}$ for all the bands. The results are shown in Figure 2, which demonstrates that quasars at $z>4$ can be well distinguished from field stars by the observation using Kiso Schmidt telescope.

TABLE 1. Observed Quasars

\begin{tabular}{lccll}
\hline Object & $\alpha$ & \multicolumn{1}{c}{$\delta$} & $\mathrm{z}$ & $\mathrm{r}$ \\
\hline PC1247+3406 & $12^{h} 49^{m}$ & $+33^{\circ} 50^{\prime}$ & 4.897 & 20.4 \\
PSS0248+1802 & $2^{h} 49^{m}$ & $+18^{\circ} 3^{\prime}$ & 4.43 & 18.4 \\
PC0751+5623 & $7^{h} 56^{m}$ & $+56^{\circ} 19^{\prime}$ & 4.281 & 19.9 \\
PC0307+0222 & $3^{h} 10^{m}$ & $+2^{\circ} 31^{\prime}$ & 4.379 & 20.4 \\
\hline
\end{tabular}

\section{Performance Test by Model SED of Quasars}

The model colors of quasars of various redshifts were calculated using simulated spectral energy distribution (SED) of quasars. We used a composite SED of quasars constructed from HST data by Zheng et al. (1997) as a SED at the rest frame. SED's for various redshifts were derived by redshifting the SED from the rest frame. The correction of Lyman absorption due to intergalactic HI clouds were executed. The intergalactic Lyman absorption as a function of redshift and wavelength were calculated from the estimation of the optical depth due to Lyman line and Lyman continuum by Yoshii and Peterson (1994). Figure 3 shows the Lyman absorption as a function of the rest frame wavelength for various redshifts. The colors of quasars of various redshits were calculated by integrating model SED's by convoluting response function of 1K-CCD or 2K-CCD systems. Calibration of colors were done using the flux standard of Hynes and Latham (1975). The colors of stars of various types were also calculated from the stellar SED's in the spectrophotometric atlas of Gunn and Stryker (1983). Figure 4 shows the simulated two-color diagram $((V-R)$ vs. $(R-I))$ of redshifted quasars on 1K-CCD system. Black dots show colors of stars in the atlas of Gunn and Stryler, and blue dots indicate those of redshifted quasars calculated from the model SED's. Red dots in the figure show calculated colors of the actual quasars, PC1247+3406 $(z=4.897)$ and PC0307+0222 $(z=4.379)$. The SED of PC1247+3406 is from Schneider et al.(1991) and that 

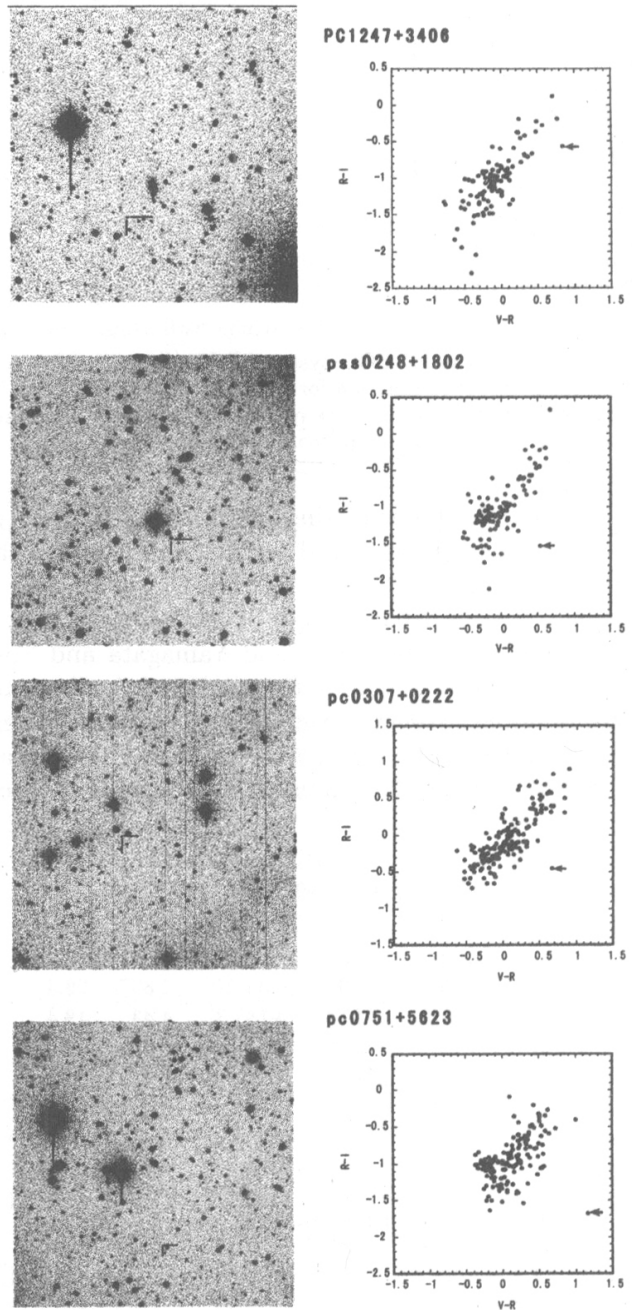

Figure 2. Left panels show $R$-band images of observed fields. Quasars are indicated by lines. Right are two-color $((V-R)$ vs. $(R-I))$ diagrams of observed images of each field. Arrows indicate quasars. These diagrams demonstrate that quasars at $z>4$ can be well distinguished from field stars by CCD color observation using the Kiso Schimidt telescope.

of PC0307+0222 is from Schneider et al.(1989). The simulated colors mimic the actual colors of quasars reasonably well. The trend of redder $(V-R)$ color with increasing redshift is shown. Smith et al.(1994) have presented similar models but for $(B-R)$ vs. $(R-i)$. Our $(R-I)$ colors of quasars with redshift $z>5$ are redder due to $R$ filter of $1 \mathrm{~K}$-CCD being slightly bluer and narrower than the standard system.

Figures $5 \mathrm{a}$ and $5 \mathrm{~b}$ show the predicted two-color diagrams on $2 \mathrm{~K}$-CCD system. Figure $5 \mathrm{a}$ is that of $(V-R)$ vs. $\left(R-I^{\prime}\right)$, and Figure $5 \mathrm{~b}$ is of $\left(R-I^{\prime}\right)$ vs. $\left(I^{\prime}-Z\right)$. As shown in Figure 5a, $(V-R)$ 


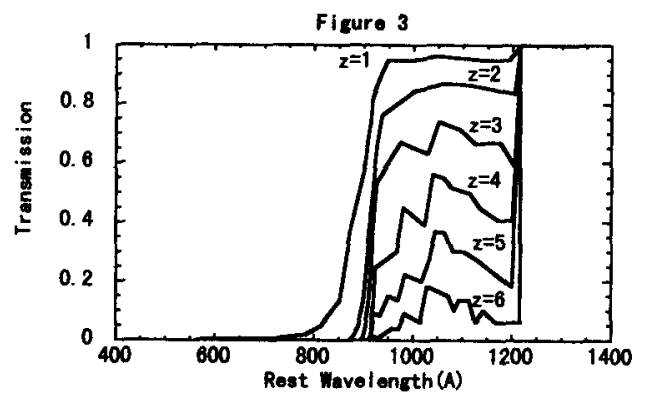

Figure 3. The Lyman absorption as a function of the rest frame wavelength for various redshifts. The intergalactic Lyman absorption as a function of redshift and wavelength were calculated from the estimation of the optical depth due to Lyman line and Lyman continuum by Yoshii and Peterson (1994).

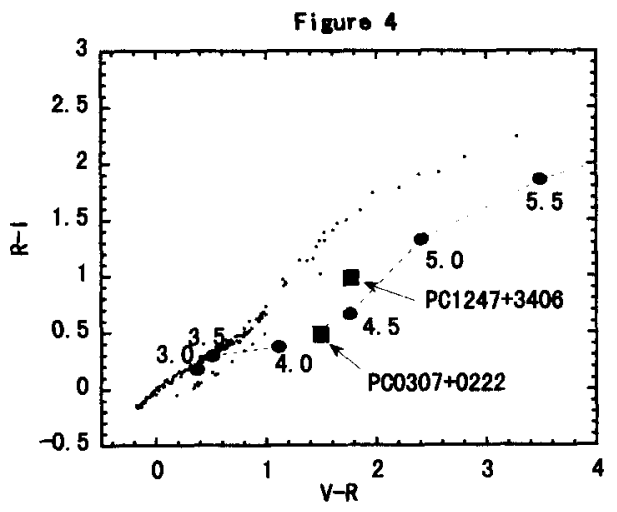

Figure 4. The simulated two-color diagram $((V-R)$ vs. $(R-I))$ of redshifted quasars on $1 \mathrm{~K}$-CCD system. Dots are colors of stars in the atlas of Gunn and Stryler(1983), and solid circles are those of redshifted quasars calculated from the model SED's. Squares in the figure indicate calculated colors of the actual quasars, PC1247+3406 $(z=4.897)$ and PC0307+0222 $(z=4.379)$. The SED of PC1247+3406 is from Schneider et al.(1991) and that of PC0307+0222 is from Schneider et al.(1989). The simulated colors mimic the actual colors of quasars reasonably well.

colors of quasars of redshift $z>5$ is greater than 3.6, therefore it is practically difficult to detect those quasars with $V$ band unless they are very bright. In this case, two-color diagram of $\left(R-I^{\prime}\right)$ vs. $\left(I^{\prime}-Z\right)$ can be used as shown in Figure 5b.

\section{Concluding Remarks}

We have verified the availability of the deep and wide-field multicolor CCD imaging method for searching high-redshift quasars using Kiso Schmidt telescope. The 2K-CCD camera system is now at the debugging stage at the Kiso observatory, and is expected to be put in service by the winter of 1997 . The survey observation will be started soon after it is available.

\section{References}

Gunn, J.E., and Stryker,L.L. (1983) $A p J S$, 52, 121

Haynes.,D.S., and Latham,D.W. (1975) $A$ pJ, 197, 593

Irwin,M., McMahon,R.G., and Hazard, C. (1991) ASPCS, 21, 117

Schneider,D.P., Schmidt, M., and Gun, J.M. (1989) AJ, 98, 1507 

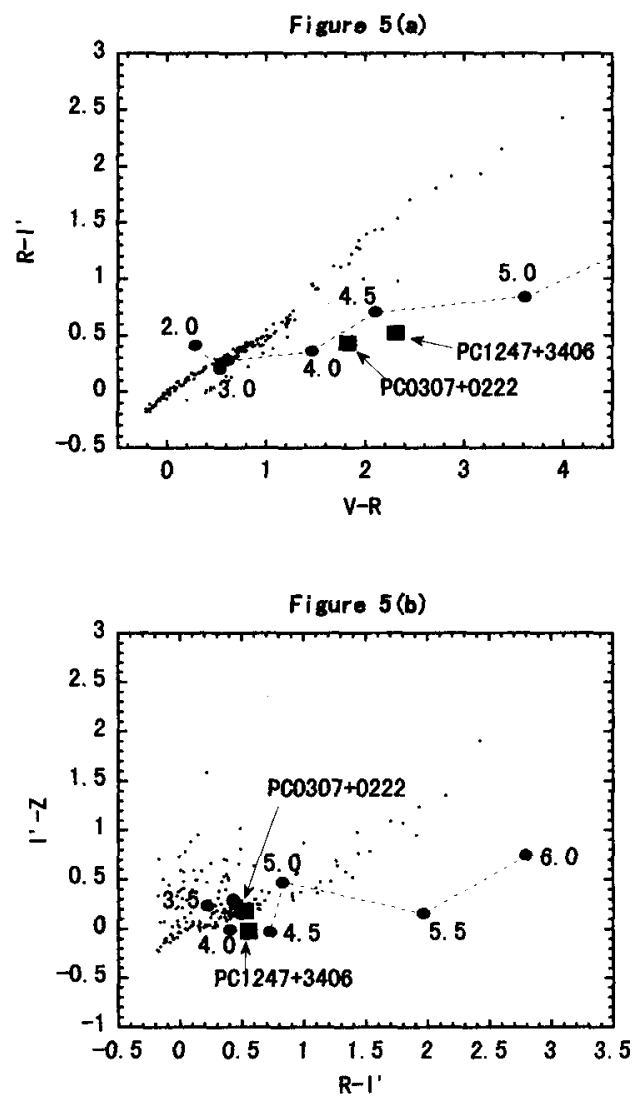

Figure 5. (a)The predicted two-color diagram of $(V-R)$ vs. $\left(R-I^{\prime}\right)$ on $2 \mathrm{~K}$-CCD system. (b)The predicted two-color diagram of $\left(R-I^{\prime}\right)$ vs. $\left(I^{\prime}-Z\right)$ on $2 \mathrm{~K}$-CCD system. Figure 5 a indicates that $(V-R)$ colors of quasars of redshift $z>5$ is greater than 3.6 , therefore it is practically difficult to detect those quasars with $V$ band unless they are very bright. In such a circumstance, two-color diagram of $\left(R-I^{\prime}\right)$ vs. $\left(I^{\prime}-Z\right)$ is useful as shown in Figure 5b.

Schneider,D.P., Schmidt, M., and Gun, J.M. (1991) $A J, 102,837$

Schneider,D.P., Schmidt,M., and Gun,J.M. (1994) $A J$, 107, 1245

Schmidt,M., Schneider,D.P., and Gun,J.M. (1995) $A J, 110,68$

Smith,J.D., Thompson,D., and Djorgovski,S. (1994) ApJ, 107, 24

Storrie-Lombardi,L.J., McMaHon, R.G., Irwin, M.J., and Hazard,C. (1996) $A p J, 468,121$

Yamagata, T. (1986) Annals Tokyo Astron. Obs., 2nd Ser., 21, 31

Yamagata, T. and Yoshii, Y. (1991) $A J, 103,117$

Yoshii,Y., and Peterson, B.A. (1994), ApJ, 436, 551

Zheng,W., Kriss,A.G., Telfer, R.C., Grimes,J.P., and Davidsen,A.F. (1997) ApJ, 475, 469 